\title{
Schade eigentlich
}

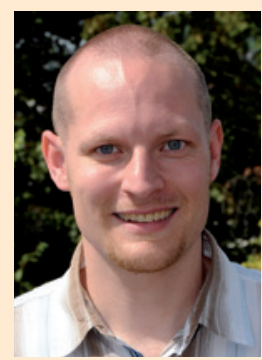

Christian Schäfer, Stuttgart
Das Wort „Transplantation“ ist in Deutschland zunehmend mit einem negativen Touch behaftet. Schade eigentlich! Denn tritt man einen Schritt zurück und hat den Blick frei auf das Ganze, erkennt man doch so viel Positives: Man bedenke beispielsweise die chirurgische Kunst, welche die Operateure beim Eingriff anwenden, und den Akt der Menschlichkeit, welcher der Organspende im Sinne des Äußerns des Willens vorausgeht, einem anderen Menschen seine eigenen Organe nach dem Tod (oder bei der Lebendspende sogar zu Lebzeiten) zur Verfügung zu stellen und ihm damit die Chance auf ein neues, längeres Leben zu ermöglichen. Auch die Organisation rund um die Organspende muss reibungslos laufen, um die Grundlage für den Erfolg einer Transplantation zu schaffen: Soll zum Beispiel nach dem Unfalltod eines potenziellen Organspenders eine Niere möglichst schnell und unversehrt zum passenden Spender gelangen, sind genau festgelegte Allokationsregeln zu befolgen. Natürlich sind hierbei der sichere Transport des Organs sowie die zeitliche Planung des gesamten Prozesses entscheidend. All dem liegen gut ausgereifte, auf dem Stand des Wissens basierende Überlegungen, gesetzliche Vorgaben und Richtlinien zugrunde. Eigentlich war alles in Butter - dachte man. In den letzten Jahren wurden allerdings Stück für Stück mutmaßliche Richtlinienverstöße einzelner Akteure in der Welt der deutschen Transplantation bekannt, die vor allem anfangs medial eine sehr große Präsenz hatten. Wen wundert es? Bei dieser hohen Sensibilität des Themas, in das auch noch die wichtige Frage „Ab wann ist der Mensch tot?" hineinspielt, ist ein Hochkochen im Zeitungsblätterwald fast unvermeidlich. Hierbei wurde allerdings auch das unglücklich gewählte und vereinfachende Wort „Organspendeskandal“ geboren. Dies hat somit den Fokus vermehrt auf den Spendeprozess gerückt - obwohl eigentlich die entdeckten Unregelmäßigkeiten bei der Warteliste auftraten. Leider hat sich als Seiteneffekt der wichtigen und notwendigen Information der Öffentlichkeit auch der Eindruck verfestigt, die gesamte deutsche „Transplantationsszene“ sei nachhaltig in dubiose Machenschaften verwickelt. Dies könnte einen großen Anteil daran haben, dass die Zahl der Organspender in den letzten Jahren zurückging.

Inzwischen ist man die Schlupflöcher im Sinne von Manipulationsmöglichkeiten der Warteliste angegangen und hat diverse Signale nach außen abgegeben (Umbau der Deutschen Stiftung Organtransplantation etc.). Insgesamt ist der Prozess der Organspende und -vergabe so sicher und transparent wie nie zuvor. Die Chance für schwarze Schafe, etwas an der Wartliste unbemerkt zu verändern, ist minimiert. Es wird auch beteuert, dass nur Einzelne für die vergangenen Unregelmäßigkeiten verantwortlich sind und keinesfalls eine groß angelegte „Szene“ existiert - was nach derzeitigem Erkenntnisstand stimmt. Leider kommt dies (noch) nicht bei der Bevölkerung an. Schade eigentlich, aber wiederum nicht verwunderlich: Zerstörtes Vertrauen in solch einem sensiblen Bereich wieder zu erlangen, ist ein mühsamer und langwieriger Prozess. Hierbei helfen sicherlich Projekte wie „Organspende macht Schule“ der Ärztekammer Westfalen-Lippe (ÄKWL) und der Techniker Krankenkassen (TK): 30 Vertreter der Ärzteschaft gehen an Schulen, um mit Schülern ab der Jahrgangsstufe 9 über die Organspende zu sprechen. Die Themen sind u.a. die Prozesse bei der Organspende und Transplantation, der Hirntod und die Verwendung von Spenderorganen - ein spannendes Vorhaben.

Auch in dieser Ausgabe der Dialyse aktuell möchten wir Aspekte der Transplantation aufgreifen: Ab Seite 423 finden Sie den Beitrag von Prof. Bernd Krüger et al., Mannheim, zum Thema „Akutes Nierenversagen und Nierentransplantation“. Dieser ist - wie die anderen Schwerpunktbeiträge zum Themenkomplex des akuten Nierenversagens (ANV) - sehr zu empfehlen. Auch kündigt der Arbeitskreis Transplantationspflege e.V. (AKTX-Pflege) sein jährliches Pflegesymposium an. Sie können sich auf Seite 408 zu der Veranstaltung am 16./17. Oktober informieren. Ich wünsche Ihnen eine angenehme Lektüre dieser Ausgabe der Dialyse aktuell! 